\title{
CuI nanoparticles on modified poly(styrene-co-maleic anhydride) as an effective catalyst in regioselective synthesis of 1,2,3-triazoles via click reaction: a joint experimental and computational study
}

Majid M. Heravi¹, Tahmineh Baie Lashaki, Hossein A. Oskooie, Tayebeh Hosseinnejad

${ }^{1}$ Department of Chemistry, Faculty of Physics \&Chemistry Alzahra University, Vanak, Tehran, Iran.

\section{Abstract}

In situ immobilization of CuI nanoparticles (NPs) on modified poly(styrene-comaleic anhydride) [SMA] was achieved. Proper immobilization of $\mathrm{CuI}$ on the prepared support was confirmed by scanning electron microscopy (SEM), energy dispersive X-ray analysis (EDAX) and inductively coupled plasma (ICP). In order to present a quantitative description for experimental features of $\mathrm{CuI} / \mathrm{SMI}$ nanocatalyst, the coordination behavior and the nature of interactions between $\mathrm{Cu}(\mathrm{I})$ ions and modified SMI ligand in gas and solution phases was computationally assessed. In this line, the mathematical properties of electron density functions were calculated and analyzed topologically via density functional theory (DFT) and quantum theory of atoms in molecules (QTAIM) approaches for different coordination modes. Encouraged by our computational results, this new catalyst was employed in a one-pot, three-component reaction involving terminal alkynes, alkyl halides and sodium azide in water resulting in highly regioselective synthesis of 1,4-disubstituted 1,2,3-triazoles in high to excellent yields. The catalyst was reused without pre-activation and recycled for at least five runs without significant decrease in its activity being observed.

\section{Keyword}

click chemistry, $\mathrm{CuI}$ nanoparticles, DFT and QTAIM computations, metal-ligand interactions, Modified poly (styrene-co-maleic anhydride). 